\title{
Spitzer-IRAC GLIMPSE of high-mass protostellar objects
}

\section{Infrared point sources and nebulae ${ }^{\star}$}

\author{
M. S. N. Kumar ${ }^{1}$ and J. M. C. Grave $e^{1,2}$ \\ 1 Centro de Astrofísica da Universidade do Porto, Rua das Estrelas, 4150-762 Porto, Portugal \\ 2 Departamento de Matemática Aplicada da Faculdade de Ciências da Universidade do Porto, Portugal \\ e-mail: [nanda;jgrave] @astro.up.pt
}

Received 15 January 2007 / Accepted 30 May 2007

\section{ABSTRACT}

\begin{abstract}
Aims. We conduct a statistical study of candidate massive protostellar objects in the 3.6-8.0 $\mu \mathrm{m}$ bands of the Spitzer Space Telescope. Methods. The GLIMPSE archive was used to obtain 3.6-8.0 $\mu \mathrm{m}$ point source photometry and images for 381 massive protostellar candidates lying in the Galactic midplane. The colours, magnitudes, and spectral indices of sources in each of the 381 target fields were analysed, and compared with the predictions of 2D radiative transfer model simulations.

Results. Infrared point sources with intrinsic reddening were found associated with several massive protostars. Although no discernable embedded clusters were found in any targets, multiple sources or associations of reddened young stellar objects were found in many sources, indicating multiplicity at birth. The spectral index $(\alpha)$ of these point sources in 3.6-8.0 $\mu$ m bands display high values of $\alpha=2-5$. A colour-magnitude analog plot was used to identify 79 infrared counterparts to the HMPOs that are bright at $8 \mu \mathrm{m}$, centred on millimetre peaks, and that display $\alpha$ values in excess of 2. Compact nebulae are found in $75 \%$ of the detected sources with morphologies that can be described well by core-halo, cometary, shell-like, and bipolar geometries similar to those observed in ultra-compact HII regions.

Conclusions. The IRAC band spectral energy distributions (SED) of the infrared counterparts of massive protostellar candidates are best described as representing YSOs with a mass range of $8-20 M_{\odot}$ in their Class I evolutionary stages when compared with $2 \mathrm{D}$ radiative transfer models. They also suggest that the high $\alpha$ values represent reprocessed star/star+disk emission that is arising in the dense envelopes. Thus we are witnessing the luminous envelopes around the protostars rather than their photospheres or disks. We argue that the compact infrared nebulae very likely reflect the underlying physical structure of the dense cores and are found to imitate the morphologies of known UCHII regions. The observations are consistent with a scenario where massive protostars have formed inside dense cores and continue to accrete matter. Our results favour models of continuuing accretion involving both molecular and ionised accretion components to build the most massive stars rather than purely molecular, rapid accretion flows.
\end{abstract}

Key words. stars: formation - ISM: HII regions - infrared: stars

\section{Introduction}

The Spitzer Space Telescope with its IRAC camera is initiating a revolution in our understanding of the star formation process by offering a great depth in sensitivity and mapping speed in the $3.6-8.0 \mu \mathrm{m}$ region of the infrared spectrum. The Galactic midplane has been mapped in these bands through the Science Legacy Program GLIMPSE. Much of the massive star formation in the galaxy occurs in its midplane; the availability of the point source photometry catalog and the image cutouts facility from the GLIMPSE programme thus give us a chance to study the statistical properties of candidate massive protostars.

In a more than decade-long effort to study the early stages of massive star formation, four major surveys to search for massive protostellar candidates have been completed that cover both the northern and southern hemispheres of the sky. These surveys are described in Molinari et al. (1996), Sridharan et al. (2002), Fontani et al. (2002), and Faúndez et al. (2004). Hereafter we will refer to them as Sri02, Mol96, Fon02, and Faun04. Followup studies of many of these targets to study the dust continuum properties have also been made (Beuther et al. 2002a;

* Figure 5 is only available in electronic form at http://www . aanda.org
Beltrán et al. 2006; Molinari et al. 2000). All these surveys are based on selection criteria using constraints on far-infrared (FIR) colours and choosing additional sign-posts of star formation, in an effort to identify phases of massive star formation prior to the onset of an ultra-compact HII (UCHII) region. The surveys are, therefore, all based on FIR and millimeter observations that can probe great depths of column density usually found in the dense cores that form massive stars. However, the large distances at which massive star-forming regions are typically found and the moderate beam sizes of FIR and millimeter telescopes used for these surveys have limited the spatial scales of observations to typically $0.1 \mathrm{pc}$ or larger. Dense cores with such physical dimensions may host a single high-mass protostellar object (HMPO) or a cluster of embedded stars. For example, using near-infrared (NIR) 2MASS data, Kumar et al. (2006) show that $25 \%$ of the HMPOs from Mol96 and Sri02 surveys are associated with embedded clusters. The Spitzer-IRAC 3.6-8.0 $\mu \mathrm{m}$ observations from the GLIMPSE survey provides an extra edge over the previous FIR and $\mathrm{mm}$ observations to probe into smaller spatial dimensions. At a comparable spatial resolution to the 2MASS data, the $8 \mu \mathrm{m}$ GLIMPSE data can penetrate extinction depths approximately four times better than the 2MASS $\mathrm{K}$ band data, assuming sensitivity limits of 14 and $12 \mathrm{mag}$ from 2.2 and $8 \mu \mathrm{m}$ 
and using the measurement that $A_{8} \mu \mathrm{m} / A_{\mathrm{K}}=0.5$ (Flaherty et al. 2007). The full-width half maximum of the Spitzer-IRAC point spread function is $\sim 2.4^{\prime \prime}$, which at a distance of $5 \mathrm{kpc}$ will trace a projected size of $\sim 10000 \mathrm{AU}$. This is typically the size of an extended envelope or a toroid around an HMPO.

In this article (Paper I), we present a statistical analysis of the mid-infrared (MIR) properties of about 381 HMPO candidates, based on the publicly available GLIMPSE database. We aim to identify the infrared counterparts of the millimeter cores, evaluate their colour properties, and search for embedded clusters associated with the HMPO targets. The emission from disk and envelope regions around young stellar objects (YSO) are known to deviate significantly from that of a normal star in the $3.6-8.0 \mu \mathrm{m}$ bands, thereby allowing the YSO classification into various evolutionary stages. While the millimeter observations are more useful for estimating the mass of the dense cores in which HMPOs are born, the GLIMPSE data will enable us to constrain the evolutionary stages and mass ranges of any point sources associated with HMPOs. In Paper II (Grave \& Kumar 2007), we will present an analysis of various physical parameters of well-identified individual sources derived from modelling the infrared-millimeter spectral energy distribution using radiative transfer models.

\section{Data selection and analysis}

Our sample of objects was derived from the four surveys of HMPOs as listed in Table 1. An estimate of the FIR luminosity and distance is available for most of the targets within the usual uncertainty factors. A distance estimate is missing for about 60 targets from Mol96, and those in Fon02 are not available from the original papers, of which about 20 targets were detected in GLIMPSE. For these targets, we assumed a fiducial mean distance of $5 \mathrm{kpc}$. As evident from Table 1, data in the GLIMPSE catalog was available for $\sim 80 \%$ of all the targets. We used both the GLIMPSE point source catalog (highly reliable) and the GLIMPSE point source archive (less reliable and more complete) to search for available data. Photometric data in the four IRAC bands at 3.6, 4.5, 5.6, and $8.0 \mu \mathrm{m}$ were obtained centred on each target.

For the purpose of cluster detection, point source data were extracted in a $600^{\prime \prime}$ diameter circle centred on each target and a detection in any one of the four bands was considered as a source. For the purpose of identifying infrared point sources and analysing their spectral properties, data were extracted from a region of 100" radius centred on each target with the constraint that the source be detected in at least 2 of the four bands with a minimum of $3 \sigma$ detection in each of the four bands. There is one important caveat in using the point source photometry catalog. Several HMPO candidates are very bright in the IRAC bands (particularly in the $8 \mu \mathrm{m}$ band) resulting in a saturated signal on the images and null data in the point source catalog. Such objects may indeed be the best counterparts of the HMPO targets in the list but will not be found in our analysis. However, these sources can be seen on the actual images where a saturated bright redstar and/or a compact nebulae can be found associated with FIR and millimeter peaks.

\section{Clustering around HMPO targets}

The point source data extracted in a 600" circle around each target was Nyquist-binned with bin sizes of $60^{\prime \prime}$ and $120^{\prime \prime}$, and contours of $2 \sigma$ and above were plotted to identify any clustering around each target. Data was available for 381 out of
Table 1. Target statistics.

\begin{tabular}{lrrr}
\hline \hline $\begin{array}{l}\text { Name of the } \\
\text { survey }\end{array}$ & $\begin{array}{r}\text { Number of } \\
\text { targets }\end{array}$ & $\begin{array}{r}\text { Number found } \\
\text { in GLIMPSE }\end{array}$ & $\begin{array}{r}\text { Number of } \\
\text { nebulae }\end{array}$ \\
\hline Molinari et al. (1996) & 161 & 79 & 59 \\
Sridharan et al. (2002) & 69 & 48 & 48 \\
Fontani et al. (2002) & 133 & 110 & 76 \\
Faúndez et al. (2004) & 146 & 144 & 105 \\
\hline Overall statistics & 509 & 381 & 288 \\
\hline
\end{tabular}

500 targets derived from all of the four surveys for which star count density enhancements were checked. No significant clusters were detected around any of the targets, although weak associations emerged when point sources only in the $5.8 \mu \mathrm{m}$ band were considered. Given the inconsistency of such weak detections depending upon the photometric band used to obtain the point sources, we conclude a null result for cluster detections for the targets surveyed. In contrast, using a similar technique with the 2MASS point source catalog, Kumar et al. (2006) were able to detect 54 clusters from the Mol96 and Sri02 samples, for all the targets that fell away from the Galactic midplane. Kumar et al. (2006) survey could not find any clusters in the Galactic midplane owing to the high extinctions in this region. While the GLIMPSE survey could easily probe the Galactic mid-plane and found several infrared counterparts and nebulae, which will be discussed in following sections, the lack of cluster detection is due to a reduced sensitivity to lower mass stars and an increased contamination of background and foreground star counts. The GLIMPSE survey data is only sensitive to $2-4 M_{\odot}$ pre-mainsequence stars at a distance of $3 \mathrm{kpc}$ owing to the short exposure times of $2 \mathrm{~s}$ in each photometric band. Embedded clusters around massive stars are mostly composed of lower mass components that will not be detected at this sensitivity. Therefore the null result here only implies the absence of major embedded clusters with massive members and/or ensembles of several hundred members.

\section{Point sources associated with HMPO targets}

Although no prominent clusters were detected in the target fields, a large number of point sources were detected in each target field of $100^{\prime \prime}$ radius with good detections in all four bands. The $100^{\prime \prime}$ limit on the radius was chosen because the dust continuum emission, mapped in the millimeter wavebands generally display emission restricted to this limit indicating that the cores/clumps are fairly compact around the IRAS sources. Thus, any MIR point sources within these limits may be associated with such dense cores/clumps forming the massive protostars. It is important to note that all four surveys have selected HMPO candidates as "isolated luminous IRAS point sources" meaning that the FIR emission is localised and point-like. Also, almost all these targets are situated away from major HII regions and in some cases at the boundaries or outskirts of well-known HII regions. Therefore, while some of the targets may be contaminated with lower mass younger protostars, most infrared point sources found in the target fields are likely candidates for intermediate or massive protostars. In the following we use the colour-colour and colour-magnitude analysis to converge on candidate massive protostars. 

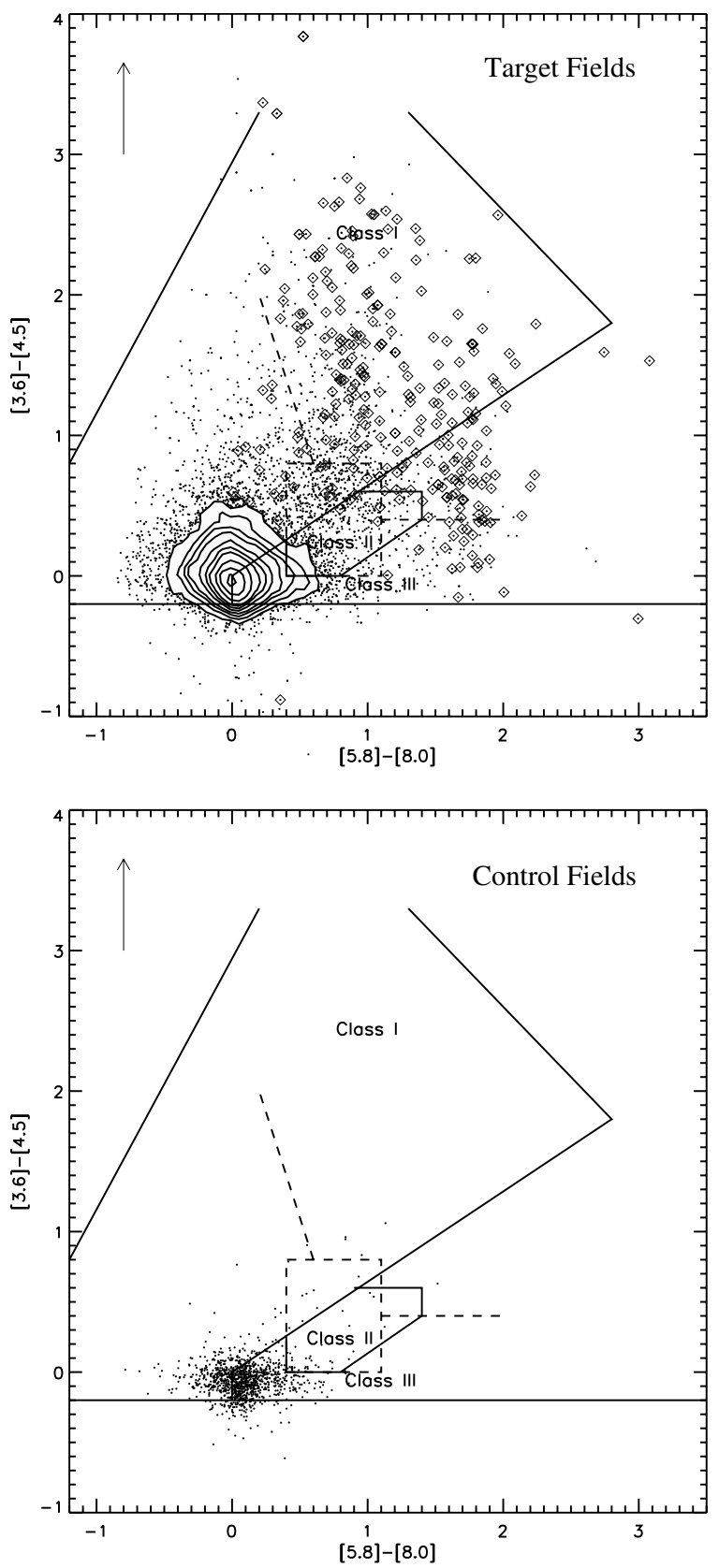

Fig. 1. Top panel: colour-colour diagram of IRAC point sources associated with candidate massive protostars. Contours are used to better display the density of points at it highest values near $(0,0)$. The box enclosed by dashed lines shows the "disk domain" and the dashed lines show the domain of embedded young stellar objects. The arrow shows a reddening vector of length $A_{\mathrm{K}}=5 \mathrm{mag}$ according to Indebetouw et al. (2005). The solid lines mark the regions occupied by various evolutionary-stage YSOs according to the models of Robitaille et al. (2006). The Class I, II, and III zones are marked. Bottom panel: same as above but for the data from 40 control fields placed $0.5^{\circ}$ adjacent to randomly selected target fields from the four surveys.

\subsection{Colours and spectral indices}

Figure 1 (top panel) shows a [3.6-4.5] vs. [5.6-8.0] colourcolour diagram for all the sources detected from all target lists. The main concentration of points at $(0,0)$ represents photospheres and higher values on both $x$ and $y$ axes represent YSOs considered to be at different evolutionary stages. The regions typical of sources at different evolutionary stages such as I, II, and III based on the 2D radiative transfer model data of Robitaille et al. (2006, hereafter RWIWD06) are shown as well. It can be seen that there are sources occupying regions representative of all the three classes, namely I, II, III with a significant fraction inside the Class I and II zones. The candidate massive protostars are based on a selection criteria that is described in Sect. 4.2.

The bottom panel in Fig. 1 shows the colours of point sources extracted from a total of 40 control fields; with 10 random fields, located 0.5 degrees adjacent to the target fields in each of the survey lists. The control fields were similar in area to the target fields with a radius of $100^{\prime \prime}$. It can be noted that the sources in these control fields represent mostly pure photospheres, except for some contamination in the Class II and Class III zones. The Class I zone is empty in the control field plot. The contamination in the Class II and III zones in the control fields could be because some of the HMPO targets are located in the vicinity of larger HII regions that can contain infrared excess sources. However, the highest infrared excess sources such as Class I objects are only associated with the target fields that are known to encompass dense cores with an embedded luminous FIR source.

Although the colour-colour diagram is useful to identify infrared excess emission of the observed targets, a quantitative measure is to compute the spectral index $\alpha$ of these sources in the observed bands. By using a simple least-square linear fit to the observed spectral energy distribution, we computed the spectral index $\alpha$ in the four IRAC bands for each point source. Figure 2 shows a histogram of the relative distribution of $\alpha$ values for the point sources associated with the massive protostellar targets. A similar distribution obtained for Spitzer-IRAC sources in the IC 348 cluster and for the YSOs in the Orion A cloud are shown for comparison. For example, in the nearby embedded cluster IC 348 (Lada et al. 2006, 3 Myr old low mass cluster), the $\alpha$ values are less than 2 because the sources are less embedded and relatively more evolved at Class II or III stages. In the Orion A cloud (1 Myr old and more massive cluster), the YSOs (note that the photospheres are removed for clarity) show $\alpha$ relatively higher than IC 348 sources. The YSOs in Orion are more embedded and are relatively younger at Class I and II stages in Orion A. The point sources associated with the massive protostellar candidates show $\alpha$ values between 2 and 5 and some even at 6 . Such high $\alpha$ sources have been observed in a wide field study of the massive star-forming region DR21/W75N where the high $\alpha$ sources were found to be well-correlated with the location of HMPOs and/or UCHII regions (Kumar et al. 2007). Thus the $\alpha$ values serve as a good indicators of the density of the surrounding medium in which the sources are embedded. Deeply embedded Class I sources are also known to produce high $\alpha$ values, which has been observed in the Serpens and Orion regions (Megeath et al. 2007). We discuss the relevance of high $\alpha$ values for this particular case in Sect. 6 .

It is evident from the results above that the spectral index is only representative of the slope of the spectral energy distribution that can be produced by point sources of different luminosities. In fact a low mass young stellar object viewed through a column of high optical depth can produce a large spectral index if detected with sufficient sensitivity. The interest in this study is to identify massive protostellar candidates. For a target situated at a fixed distance and viewed through a fixed extinction, it is the brightness or luminosity of the source that identifies the massive objects. Therefore a colour-magnitude diagram is needed to isolate sources with high luminosity, as well as large infrared excess. 


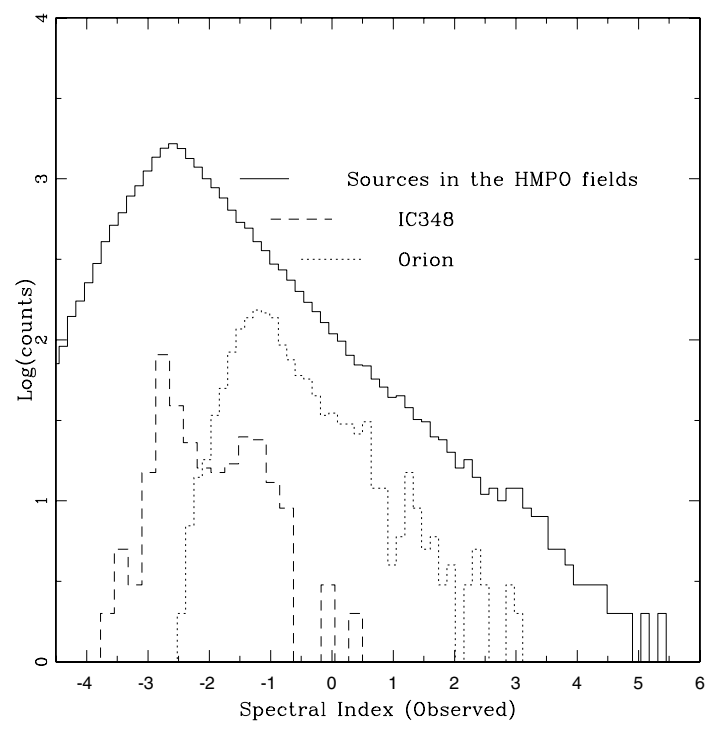

Fig. 2. Histogram of the observed spectral indices $(\alpha)$ for sources associated with candidate massive protostellar candidates. The dotted curve shows a similar histogram for the IRAC YSOs in Orion A cloud (Megeath et al. 2007) and the dashed curve shows the distribution of sources in IC348 (Lada et al. 2006).
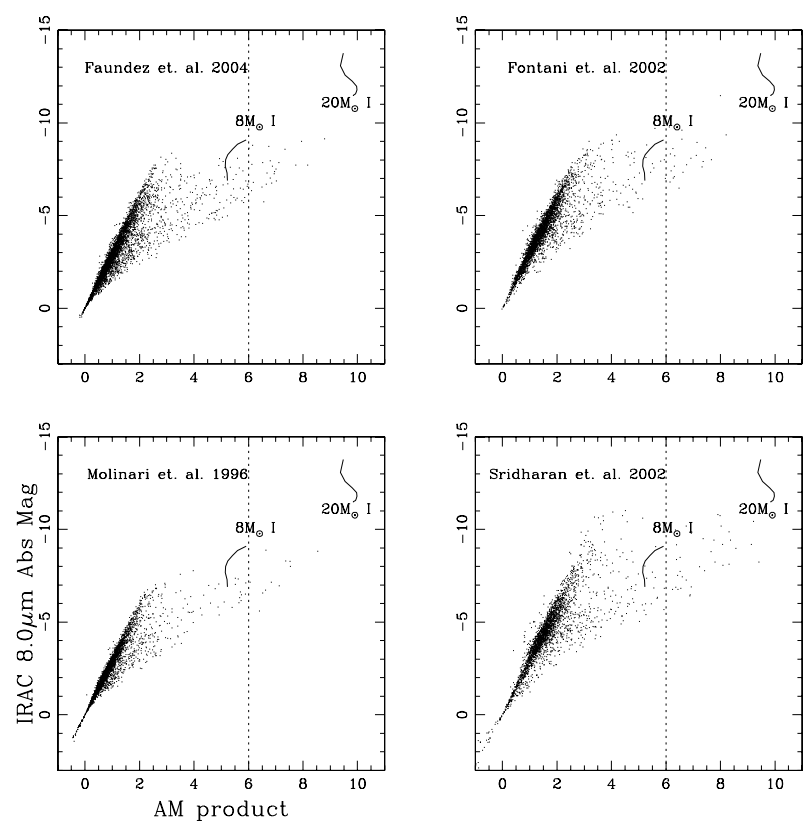

Fig. 3. The AM product plotted versus the [8.0] $\mu \mathrm{m}$ absolute magnitude for the sources detected from the four surveys of massive protostellar candidates. The solid curves represent $20 M_{\odot}$ and $8 M_{\odot}$ class I object model curves for all inclinations computed by RWIWD06.

\subsection{Spectral index vs. magnitude diagrams}

The sources in our target list are at different distances and a comparison of colours and magnitudes is possible only by correcting for distance and line-of-sight extinction. The distances to individual targets and luminosities were obtained from the respective survey papers listed in Table 1 . However, the line-of-sight extinction to each source is not easily available, and its estimation can vary depending upon the method and wavelength used to make the extinction measurement. We therefore applied only the distance correction and converted the photometric magnitudes to absolute magnitudes for each source using the appropriate distance. Since the extinction correction is not made and the IRAC [5.8-8.0] colour shows no difference for a reddened object (note that the reddening vector is vertical) some luminous reddened background giants can contaminate the sample. However, these contaminations can decrease when objects are selected through the AM product criteria described below, since the spectral index as a whole enters into the AM product.

Next, we found that a simple colour magnitude diagram is confusing because of a large scatter of points around the region occupied by main-sequence stars. For example, the scatter due to observational errors and different line-of-sight extinction shows a significant spread at $(0,0)$ in the colour-colour diagram shown in Fig. 1 which translates to a spread of similar width on a colour-magnitude diagram. To reduce this scatter effect and to separate more effectively the luminous members, we define a product called the "AM product" (alpha-magnitude product), meaning the product of the spectral index $\alpha$ and absolute magntidue for a source. For convenience of plotting, we define $\mathrm{AM}=-M_{8} \mu \mathrm{m} \times(\alpha+6) / 10$ where $M_{8} \mu \mathrm{m}$ is the $8.0 \mu \mathrm{m}$ absolute magnitude of the source and $\alpha$ the observed spectral index. The constants 6 and 10 in the above equation were chosen arbitrarily to effectively separate the high $\alpha$ sources from the rest. For example a source of $M_{8} \mu m=-5$ with $\alpha$ values of 0 and 3 will have an AM product of 3 and 4.5. Recall the necessity of computing an AM product to obtain luminous sources along with their high colours rather than separating due to colours alone as explained early in this section. Figure 3 shows AM vs. $8.0 \mu \mathrm{m}$ absolute magnitude plots for the sources associated with targets from the four different surveys. Two model curves for an $8 M_{\odot}$ and a $20 M_{\odot}$ Class I objects from RWIWD06 are shown in this figure for comparision with the observed points. The model curves represent the locus of points with a particular mass and for all inclination angles.

It can be seen from Fig. 3 that while most points are concentrated around the dense slanting branch representative of photospheres, a significant fraction occupy the zones representative of young stellar objects. The area encompassed by the curves representing Class I sources of $8 M_{\odot}$ and $20 M_{\odot}$ are well separated from the rest of the points, suggesting the presence of massive protostars. All sources with an AM product higher than 6 in Fig. 3 are considered as candidate infrared counterparts to HMPO targets. A total of 79 point sources with photometry available in all four IRAC bands could be classified as HMPO targets. The number of HMPOs detected in Mol96, Sri02, Fon02, and Faun04 are 11, 27, 23, and 25, respectively. This count does not include the brightest counterparts, which are saturated in the IRAC bands. More rigorous isolation of HMPO sources using measurements at other wavelengths and constructing SEDs over a wider wavelength range will be discussed in Paper II. Figure 4 shows a spatial plot of the high AM product sources with respect to the IRAS PSC central coordinates for each target field. It can be seen that, while there is some spread of these sources within an $100^{\prime \prime}$ box, most sources with high AM values are centred on the IRAS peaks in the target fields. The associated millimeter dust continuum peaks also show some scatter with respect to IRAS centres (e.g. Beuther et al. 2002a). The observed scatter is consistent with the spread of the centres of the $1.2 \mathrm{~mm}$ peaks from the respective IRAS positions. Thus, the correlation between the IRAS centres and the IRAC-point sources with high AM products suggest that we are indeed observing some of the MIR counterparts of the candidate massive protostars.

In summary, most of the high AM sources in the Class I region of Fig. 1 are very good candidates, although the high AM sources with $[5.8-8.0]<0.4$ colours could also be reddened 


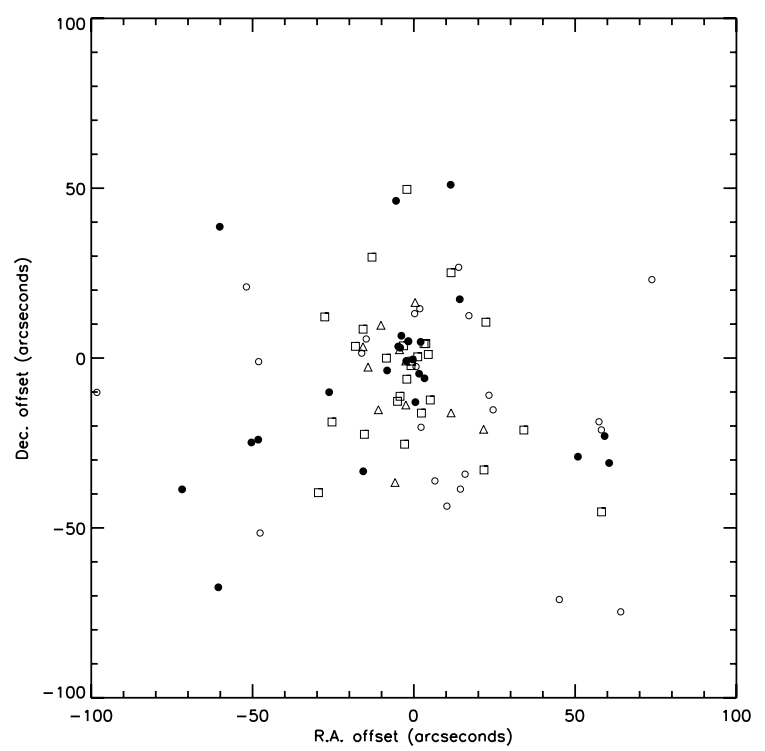

Fig. 4. Spatial distribution of high AM sources with respect to the central coordinates of the associated IRAS sources. Targets from all the four surveys, namely Mol96 (triangles), Sri02 (squares), Fon02 (open circles), and Faun04 (filled circles), are plotted. Note that bright saturated targets, which comprise a majority of high AM sources, do not appear on this plot.

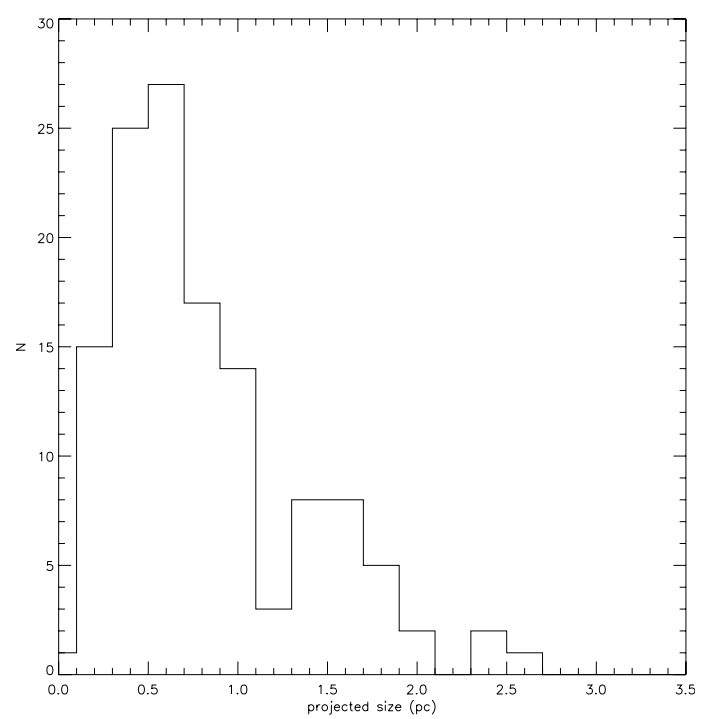

Fig. 6. Histogram of projected sizes of the infrared nebulae.

photospheres. Some of the candidates are probably evolved protostars and some can imitate red colours due to nebular contamination; therefore, a complete SED analysis will be used to remove such confusions in Paper II.

\section{Compact nebulae}

As mentioned in Sect. 2, many infrared counterparts to HMPOs can be very bright in the IRAC bands saturating the detectors, resulting in null data in the photometry catalog. Also, interesting nebulae around HMPOs cannot be analysed using point source photometry catalog. We therefore used the image cutouts facility available on the IRSA webpage to retrieve IRAC images of size $300^{\prime \prime}$ centred on each target. These images reveal compact nebulae $\left(10^{\prime \prime}-60^{\prime \prime}\right.$ angular sizes) around several targets. Detection statistics of these nebulae are listed in Table 1. The nebulae are found to be brightest in the $8 \mu \mathrm{m}$ band and mostly invisible in the $3.6 \mu \mathrm{m}$ IRAC band. We have used the 4.5, 5.8 and $8.0 \mu \mathrm{m}$ band images coded as blue, green, and red, respectively to generate composite false colour images for the observed nebulae. The false colour images for Sridharan et al. (2002) targets are shown in Fig. 5 (available online) ${ }^{1}$. In these figures large circlular symbols represent sources with AM values higher than 6 and small circles represent sources with AM values between 4 and 6 . In some cases, although the circular symbols are absent, a bright saturated star can be found at the centre of the field, which corresponds to the massive protostars. In Paper II, these sources will be modelled using data from 2MASS and mm bands as well.

Among the four target samples, Sri02 sample is supposed to represent the youngest HMPO candidates as that sample used a criteria to select targets without any significant radio continuum emission. Mo196 and Fon02 samples are known to have a mixture of source with and without significant radio continuum emission, while Faun04 list contains several wellknown UCHII regions. Although most of the nebulae in our figures are restricted to the extracted $300^{\prime \prime}$ region, the nebulae in Sri02 that represent the youngest sample are relatively more compact. A careful examination of Fig. 5 will reveal that the nebulae associated with HMPO targets repeatedly display cometary (e.g.: I18437-0216), core-halo (e.g.: I18337-0743, I19403+2258), shell-like (e.g.: I19198+1423), Gaussian (e.g.: I19074+0752), and bipolar (e.g.: I18530+0215, I19213+1723) morphologies. A rough size of these nebulae was measured on each $8 \mu \mathrm{m}$ image using the ruler option on the SAOIMAGE DS9 image display widget. Some images display only a single welldefined nebula, whereas others show a group of compact nebulae. In those cases where multiple components were found, we measured the mean size of the smaller components and also the upper limit of the overall size inside which the small components are embedded. A histogram of the angular scales converted to projected distances of these nebulae are shown in Fig. 6. The sizes are typically in the range $0.1-1.0 \mathrm{pc}$ with a mean value of $0.5 \mathrm{pc}$. Even the most extended nebulae projects to a maximum of $2.5 \mathrm{pc}$. Therefore the dimensions of the infrared nebulae are similar to or smaller than the size of dense cores as traced by the $1.3 \mathrm{~mm}$ continuum maps. UCHII regions display similar morphologies on high-resolution radio continuum images (Kurtz et al. 1994), and bipolar shapes are representative of outflows. The $8.0 \mu \mathrm{m}$ band is dominated by PAH emission, which is known to be a tracer of radiation temperature. Ionising radiation from young massive stars, that may not yet be strong enough to produce a significant ionised region may therefore be traced by these infrared nebulae. Indeed a recent investigation has shown that the underlying structure of the ISM in such nebulae can possibly be inferred using the morphology of the nebulae at various density regimes and ionising fluxes (Heitsch et al. 2007). Therefore, the morphology of the nebulae found here may well indicate the morphology in which ionising radiation is escaping from the underlying set up of physical structures close to the star. Recently Churchwell et al. (2006) have used the GLIMPSE images to identify bubbles around OB stars in the Galaxy and argue that the smaller bubbles around several B stars are those produced by relatively soft radiation that fails to produce significant HII regions. The nebulae presented here may well represent such bubbles or could be simple reflection nebulae due to an evolved generation of B stars.

1 The colour composites for the Mol96, Fon02, and Faun04 surveys can be accessed at http://www . astro.up.pt/ nanda/hmpo/ 


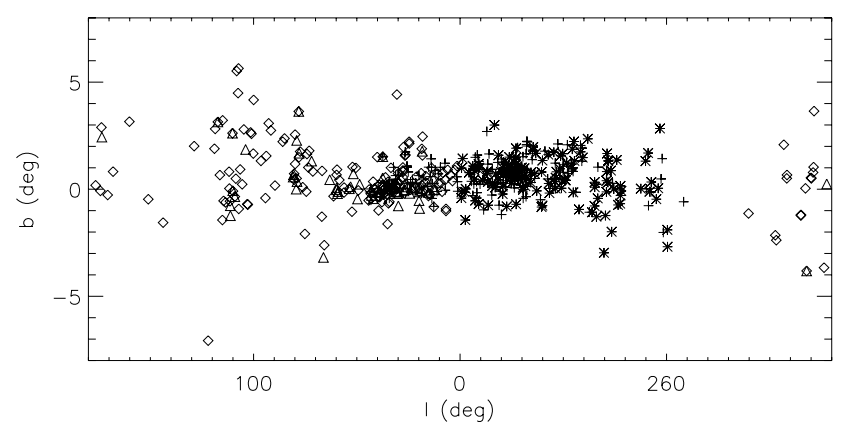

Fig. 7. Galactic positions of all the targets from Sridharan et al. (2002) (triangles), Molinari et al. (1996) (diamonds), Fontani et al. (2002) (asterisks), and Faúndez et al. (2004) (plus marks).

\section{Discussion}

All four sample sets of targets used in this study have FIR luminosities in the range $10^{3}-10^{5} L_{\odot}$ and satisfy similar FIR colour criteria. Nevertheless, the targets from the Fon02 and Faun04 surveys reveal significantly large number of nebulae and reddened sources in comparision to targets from the Sri02 and Mol96 samples. To understand these variations among the samples, we plotted the galactic positions of all targets (Fig. 7) and found that the Fon02 and Faun04 targets are concentrated in the galactic mid-plane $(b= \pm 2)$ and close to the Galactic centre ( $l=0-40$ and $l=280-360)$, whereas the Sri02 and Mol96 targets are situated relatively far away from the Galactic centre ( $l=10-220)$ and also have a wider distribution with respect to the mid-plane $(b= \pm 3)$. The Faun04 sample also contains a large fraction of well-known UCHII regions that are found in the Galactic mid-plane. The HMPO candidates from the RMS survey also show similar concentration limited to the inner plane of the galaxy (Urquhart et al. 2006). Since the Spitzer bands are well-suited to probing the midplane and most HMPO candidates are concentrated in this area, the highest number of nebulae found in the samples of Fon02 and Faun04 is not a surprise. This result also indicates that much of the ongoing massive star formation is also found within the Galactic mid-plane in accordance with the known scale height of 100pc for OB stars. The location of the largest number of GLIMPSE nebulae in the midplane also explains why Kumar et al. (2006) did not find any clusters in this region where the 2MASS $\mathrm{K}$ band suffers high extinction.

As shown in Sect. 3, not finding any clusters does not necessarily imply the absence of embedded clusters. For example, deep near-infrared imaging of the source I19343+2026 (at $0.5^{\prime \prime}$ seeing and $K=19 \mathrm{mag}$ limit) shows a rich cluster at $2 \mu \mathrm{m}$ and only a few stars in the Spitzer data. This may suggest that many of the compact nebulae found in the Spitzer data may indeed be undetected embedded clusters. In such an event, the cluster statistics of $25 \%$ derived by Kumar et al. (2006) is clearly a lower limit for the HMPO candidates. This result will have important consequences on the nature and evolutionary state of the high-mass dense cores studied so far.

The point sources detected from the GLIMPSE survey show colours and magnitudes representative of young stellar objects in the mass range $8 M_{\odot}-20 M_{\odot}$ and in the evolutionary stages between Classes I and III. The high $\alpha$ values indicate a steeply rising spectral energy distribution in the $3.6-8.0 \mu \mathrm{m}$ region and mostly show no detections in the $2 \mu \mathrm{m} K$ band from 2MASS. The radiative transfer models database from RWIWD06 was used to examine the individual components of emission for each model, namely the star, disk and envelope contributions. In these models by RWIWD06, radiation arising from each of the entities star, disk, and envelope are computed. For example, the envelope emission in these models is calculated as radiation originating in the envelope but not necessarily produced inside the envelope. Reprocessed star light for which the envelope is the starting point is treated as envelope emission. Comparing the individual model components from star, disk, and envelope, we find that the observed data in IRAC bands are best described by the envelope emission component. In most cases, the stellar photosphere and/or the accreting disk have very little or no contribution in the IRAC bands. Therefore the high $\alpha$ values in the IRAC bands is mostly due to emission arising in envelopes, which is due to reprocessed star and/or star+disk emission. In most cases, the stellar photosphere and/or the accreting disk have no directly contributing emission in the IRAC bands. Thus we are witnessing the envelopes of massive young stars through these observations. The model parameters that fit the observed data well suggest that a majority of the HMPO candidates host massive protostars in the mass range $8 M_{\odot}-20 M_{\odot}$ and beyond. More rigorous identification of individual HMPOs can be made by constructing the SED over a wider wavelength range at similar spatial resolution and modelling them with radiative transfer analysis. In Paper II we will discuss the results from such an analysis of individual massive protostars by building the SED with all available data in the literature.

Thus far, the analysis presented here indicates the presence of HMPOs identified by their shining envelopes that are embedded in compact infrared nebulae. Apart from this infrared view, we know a priori from the original survey papers of these HMPOs that they are dense cores with high column densities (Beuther et al. 2002a), ongoing infall (Fuller et al. 2005), and outflow activity (Beuther et al. 2002b; Zhang et al. 2005). High-sensitivity observations with the Very Large Array (VLA) show unresolved centimetre continuum emission associated with many of these point sources (Molinari et al. 1998; Carral et al. 1999; Zapata et al. 2006), some of which are also known to be well-identified driving sources of massive molecular outflows (Beuther et al. 2004). For example in the sample of Mol96, we found IR counterparts for 27 unresolved VLA sources within a $5^{\prime \prime}$ radius, of which most had a saturated flux at $5.8 \mu \mathrm{m}$ and $8 \mu \mathrm{m}$ bands. Six sources had data in all bands and satisfied the criteria of high AM sources, and all these six sources coincide with the VLA peaks to an accuracy of $\leq 3^{\prime \prime}$. In an effort to separate HMPOs based on centimetre continuum emission, Urquhart et al. (2006) find several MSX sources with and without radio continuum emission, implying that a large fraction of the sources indeed display centimetre continuum emission along with other signposts of HMPOs such as millimetre and FIR emission. Carral et al. (1999) found accurately matching compact $3.6 \mathrm{~cm}$ continuum sources associated with the dust emission peaks of 12 sources derived from Sri02 and Mol96 samples. Among them, four sources lie in the galactic plane covered by GLIMPSE data, and we find high AM sources in all the four sources. The complete list of sources that satisfy the HMPO criteria and their correlation with the observed centimetre flux will be discussed in Paper II. The centimetre continuum emission represent free-free emission in ionised gas and is thought to trace the ultra-compact or hyper-compact HII regions around some of the HMPOs situated inside the thick shining envelopes described above. From the available data, although it is impossible to rule out the possibility that all of this emission arises from jets, it is also improbable that the centimetre emission in all these sources is originating in jets (e.g. Zapata et al. 2006). 
If we assume that a good fraction of the observed centimetre peaks are HII components and add the result from this work that they are associated with IR counterparts satisfying the HMPO criteria along with other signposts of ongoing accretion such as outflow and disk, we are then witnessing a combination of ionised and molecular material around accreting stars. This may suggest that the accretion is ongoing and very likely composed of both molecular and ionised components of gas. The presence of ionised matter may be particularly true when the embedded star is close to $20 M_{\odot}$ or more. Ionised accretion flows are demonstrated well by the study of the UCHII region such as G10.6-0.4 (Keto 2002a) and accretion signatures in the $\mathrm{Br} \gamma$ line profiles (which can represent ionised gas) in some massive young stars (Blum et al. 2004). Combining the implications of observations from the centimetre and millimetre to the infrared thus leads us to a scenario where the dense cores are hosting precursors to OB type stars, some of which have even produced a compact ionised region and continue to accrete, adding more mass to the central star. Such a process may be of particular importance in producing $\mathrm{O}$ stars. Therefore these observations are supportive of massive star formation scenarios through a continuuing accretion process with a combination of both molecular and ionised gas components (Keto 2002b, 2003) rather than rapid accretion of molecular gas alone (McKee \& Tan 2003). Rapid accretion of molecular gas alone may be prominent only in the very early (and short time scale) class 0 phase of the HMPOs, observed in some "mm-only" type of objects (e.g. Hill et al. 2006).

\section{Summary \& conclusions}

We have used the GLIMPSE point source catalog and the image cutout facility to investigate the properties of candidate massive protostellar objects distributed over the northern and southern hemispheres of the sky. Data were found for 381 out of 500 examined targets. The analysis of the point source photometry and images can be summarised as follows:

- The GLIMPSE data could probe the HMPO targets in the Galactic midplane revealing their IR counterparts. No significant clustering was observed around HMPO targets; however, multiple components or isolated bright point sources with intrinsic reddening were found in most cases indicating multiplicity at birth.

- Colour-colour diagram analysis of point sources found in the target fields and 40 randomly selected control fields clearly demonstrate the presence of reddened Class I and II type sources lying in the target fields. The spectral index $(\alpha)$ of such point sources computed using fluxes in the IRAC bands display high $\alpha$ values of 3-5, suggesting their deeply embedded nature in dense cocoons around the HMPOs. Absolute magnitude vs alpha-magnitude (AM product) diagrams demonstrate that these point sources occupy zones that are representative of massive young stars ranging in the mass between $8-20 M_{\odot}$ or more, implying the presence of massive protostars.

- A total of 79 point sources could be classified as HMPO candidates, and they display a good spatial correlation with the associated IRAS sources.

- Nearly $60 \%$ of the targets are surrounded by compact infrared nebulae, particularly luminous in the $8 \mu \mathrm{m}$ band. These nebulae display morphologies similar to UCHII regions such as cometary, core-halo, shell, and bipolar shapes. The size distribution of these nebulae for the SriO2 and
Mo196 sample (which are assumed to be youngest subsets) display a characteristic size scale of $0.1-1 \mathrm{pc}$, with a mean value of $0.5 \mathrm{pc}$, showing that the nebulae are limited to the boundaries of the dense cores mapped by the millimetre continuum emission and may be reflecting the underlying physical structure of these cores.

- The GLIMPSE view suggests that the massive star-forming dense cores contain precursors to OB stars shining through their thick envelopes and surrounded by compact infrared nebulae. The observed correlation of unresolved centimetre continuum emission from VLA and the GLIMPSE counterparts suggests that some of these HMPOs have produced an ultra/hypercompact HII region close to the star, and they continue to accrete matter. Therefore both ionised and molecular components of accretion are likely to be involved in building the most massive stars such as $\mathrm{O}$ stars. The observations thus favour a scenario of massive star formation through continuuing accretion involving both molecular and ionised flows rather than rapid accretion of molecular gas alone.

Acknowledgements. We thank the referee Tom Megeath for useful suggestions that have improved the presentation of the paper and also for providing the Orion data for comparision. M.S.N.K. and J.M.C.G. are supported by a research grant POCTI/CFE-AST/55691/2004, and J.M.C.G. is supported by a doctoral fellowship SFRH/BD/21624/2005 approved by FCT and POCTI, with funds from the European community programme FEDER. This research made use of the NASA/ IPAC Infrared Science Archive, which is operated by the Jet Propulsion Laboratory, California Institute of Technology, under contract with the National Aeronautics and Space Administration.

\section{References}

Beltrán, M. T., Brand, J., Cesaroni, R., et al. 2006, A\&A, 447, 221 Beuther, H., Schilke, P., Menten, K. M., et al. 2002a, ApJ, 566, 945 Beuther, H., Schilke, P., Sridharan, T. K., et al. 2002b, A\&A, 383, 892 Beuther, H., Schilke, P., \& Gueth, F. 2004, ApJ, 608, 330

Blum, R. D., Barbosa, C. L., Damineli, A., Conti, P. S., \& Ridgway, S. 2004, ApJ, 617, 1167

Carral, P., Kurtz, S., Rodríguez, L. F., et al. 1999, Rev. Mex. Astron. Astrofis., 35,97

Churchwell, E., Povich, M. S., Allen, D., et al. 2006, ApJ, 649, 759

Faúndez, S., Bronfman, L., Garay, G., et al. 2004, A\&A, 426, 97 (Faun04)

Flaherty, K. M., Pipher, J. L., Megeath, S. T., et al. 2007, ApJ, 663, 1069

Fontani, F., Cesaroni, R., Caselli, P., \& Olmi, L. 2002, A\&A, 389, 603 (Fon02)

Fuller, G. A., Williams, S. J., \& Sridharan, T. K. 2005, A\&A, 442, 949

Grave, J. M. C., \& Kumar, M. S. N. 2007, Paper II, in preparation

Heitsch, F., Whitney, B. A., Indebetouw, R., et al. 2007, ApJ, 656, 227

Hill, T., Thompson, M. A., Burton, M. G., et al. 2006, MNRAS, 368, 1223

Indebetouw, R., Mathis, J. S., Babler, B. L., et al. 2005, ApJ, 619, 931

Keto, E. 2002a, ApJ, 568, 754

Keto, E. 2002b, ApJ, 580, 980

Keto, E. 2003, ApJ, 599, 1196

Kumar, M. S. N., Keto, E. R., \& Clerkin, E. 2006, A\&A, 449, 1033

Kumar, M. S. N., Davis, C. J., Grave, J. M. C., Ferreira, B., \& Froebrich, D. 2007, MNRAS, 374, 54

Kurtz, S. E., Churchwell, E., \& Wood, D. O. S. 1994, ApJS, 91, 659

Lada, C. J., Muench, A. A., Luhman, K. L., et al. 2006, AJ, 131, 1574

McKee, C., \& Tan, J. 2003, ApJ, 566, 931

Molinari, S., Brand, J., Cesaroni, R., \& Palla, F. 1996, A\&A, 308, 573 (Mol96)

Molinari, S., Brand, J., Cesaroni, R., Palla, F., \& Palumbo, G. G. C. 1998, A\&A, 336,339

Molinari, S., Brand, J., Cesaroni, R., \& Palla, F. 2000, A\&A, 355, 617

Megeath, S. T., et al. 2007, in preparation

Robitaille, T. P., Whitney, B. A., Indebetouw, R., Wood, K., \& Denzmore, P. 2006, ApJSS, 167, 256 [RWIWD06]

Sridharan, T. K., Beuther, H., Schilke, P., Menten, K. M., \& Wyrowski, F. 2002, ApJ, 566, 931 (Sri02)

Urquhart, J. S., Busfield, A. L., Hoare, M. G., et al. 2007, A\&A, 461, 11

Zapata, L. A., Rodríguez, L. F., Ho, P. T. P., Beuther, H., \& Zhang, Q. 2006, AJ, 131,939

Zhang, Q., Hunter, T. R., Brand, J., et al. 2005, ApJ, 625, 864 
M. S. N. Kumar and J. M. C. Grave: Spitzer-IRAC GLIMPSE of high-mass protostellar objects. I., Online Material p 1

\section{Online Material}


M. S. N. Kumar and J. M. C. Grave: Spitzer-IRAC GLIMPSE of high-mass protostellar objects. I., Online Material p 2

Sridharan 2002
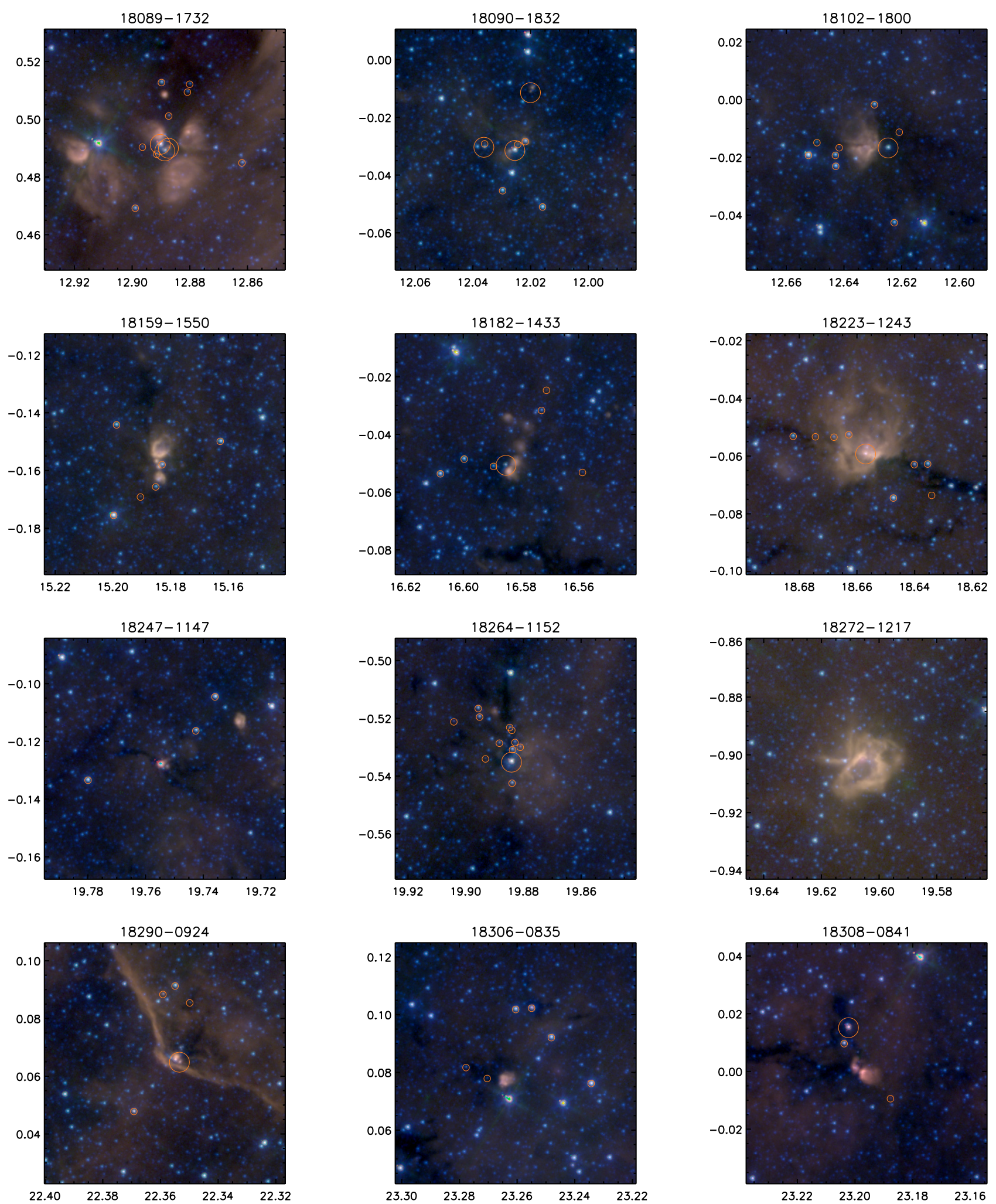

Fig. 5. Three colour composites of the targets found from Sridharan et al. (2002) survey. The $4.5 \mu \mathrm{m} 5.8 \mu \mathrm{m}$ and $8 \mu \mathrm{m}$ channels are coded blue, green and red respectively. Large circles represent sources with $\mathrm{AM}>6$ and small circles show sources with $4<\mathrm{AM}<6$. 
M. S. N. Kumar and J. M. C. Grave: Spitzer-IRAC GLIMPSE of high-mass protostellar objects. I., Online Material p 3 Sridharan 2002
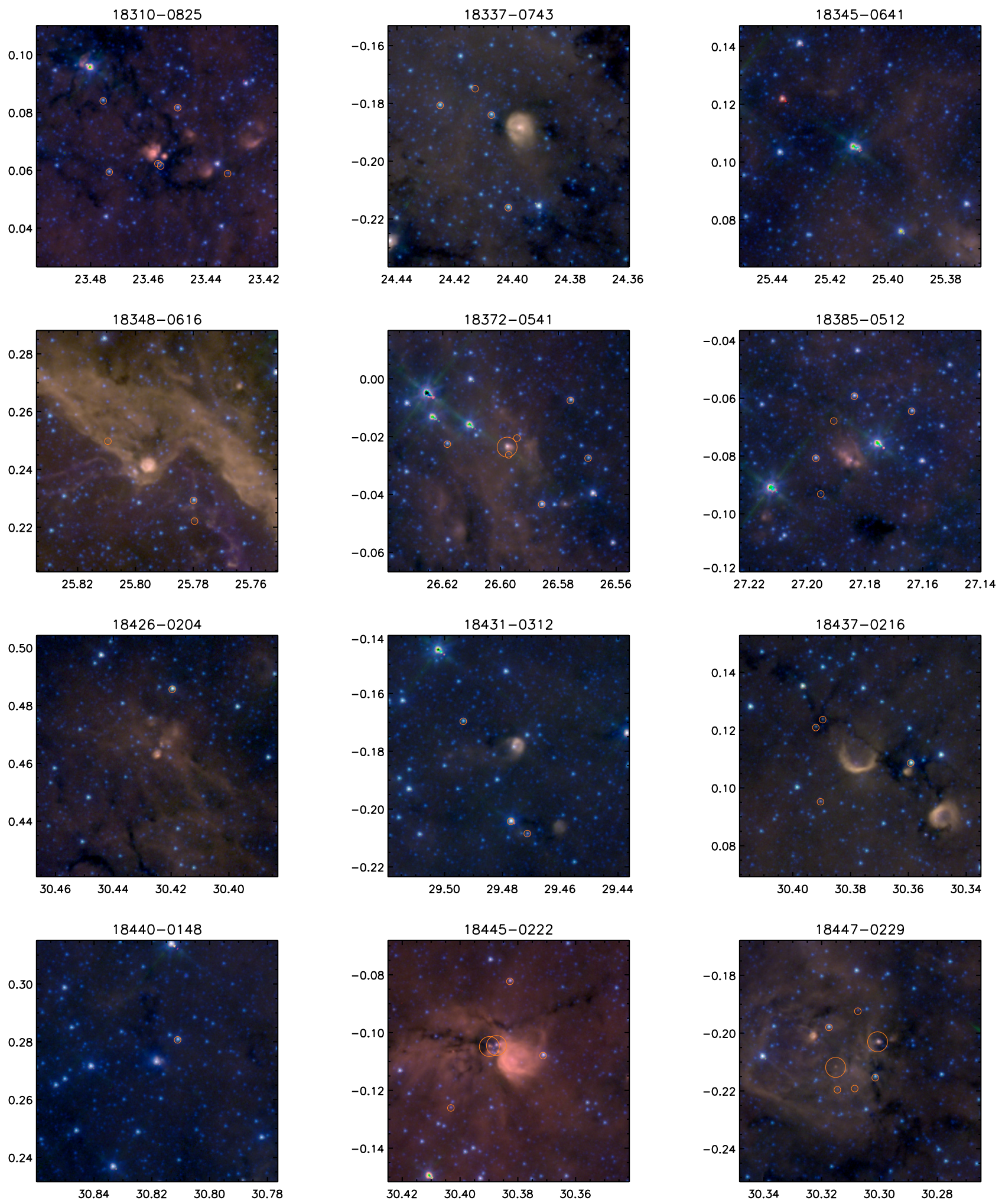

Fig. 5. continued. 
M. S. N. Kumar and J. M. C. Grave: Spitzer-IRAC GLIMPSE of high-mass protostellar objects. I., Online Material p 4 Sridharan 2002
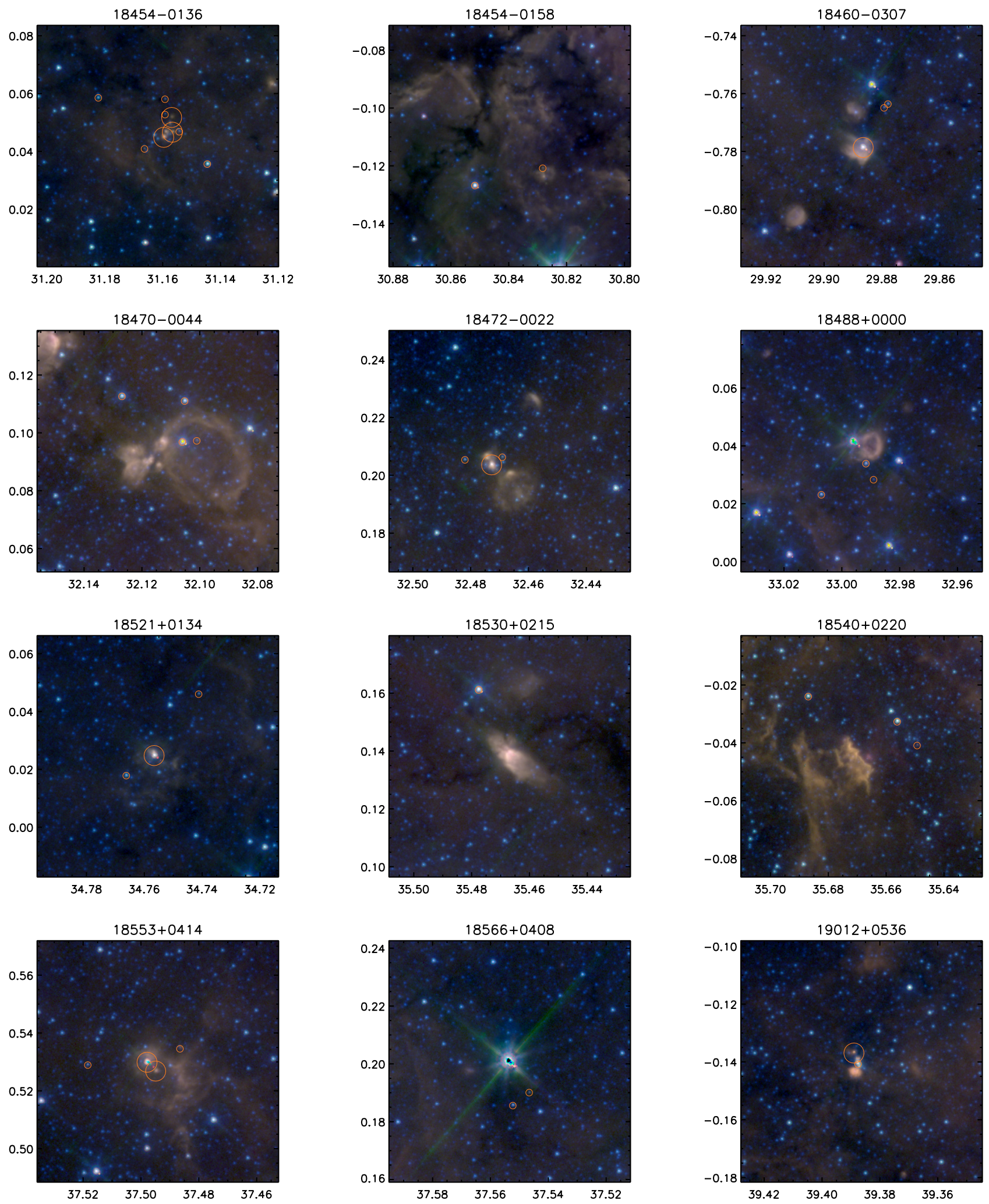

Fig. 5. continued. 
M. S. N. Kumar and J. M. C. Grave: Spitzer-IRAC GLIMPSE of high-mass protostellar objects. I., Online Material p 5

Sridharan 2002
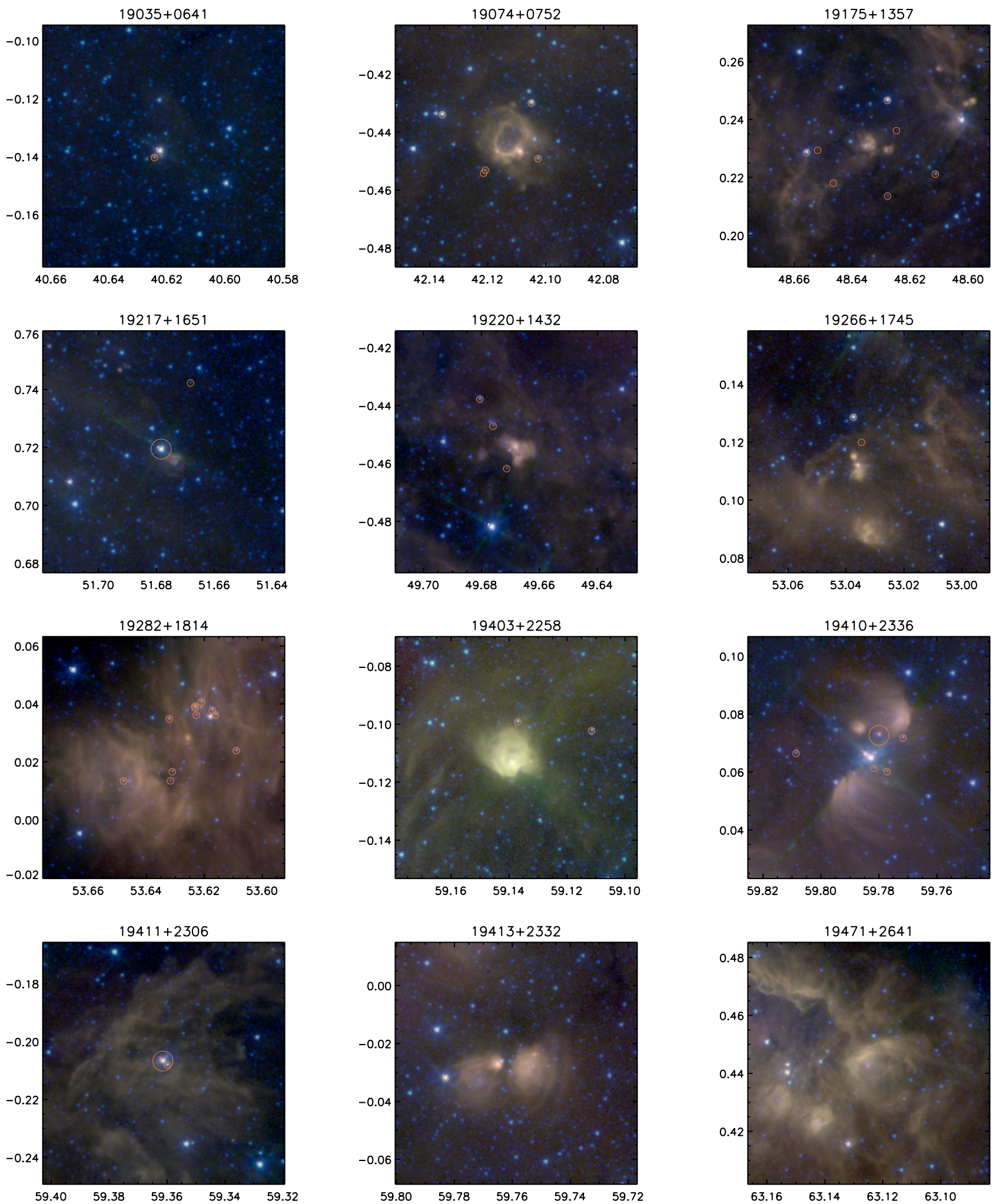

Fig. 5. continued. 